\title{
Reasons influencing the preferences of prospective patients and orthodontists for different orthodontic appliances
}

\author{
Guido Artemio \\ Marañón-Vásquez ${ }^{\mathrm{a}}$ (1) \\ Luísa Schubach \\ da Costa Barreto ${ }^{a}$ \\ Matheus Melo Pithon ${ }^{b}$ \\ Lincoln Issamu Nojima ${ }^{a}$ \\ Matilde da Cunha \\ Gonçalves Nojima ${ }^{a}$ \\ Mônica Tirre \\ de Souza Araújo ${ }^{a}$ \\ Margareth Maria \\ Gomes de Souza ${ }^{\mathrm{a}}$ (1)
}

${ }^{a}$ Department of Pediatric Dentistry and Orthodontics, School of Dentistry, Federal University of Rio de Janeiro, Rio de Janeiro, Brazil

${ }^{b}$ Department of Health 1, School of Dentistry, Southwest Bahia State University, Bahia, Brazil

\begin{abstract}
Objective: To evaluate the reasons influencing the preferences for a certain type of orthodontic appliance over another among prospective patients (PP) and orthodontists. Methods: A total of 49 PP and 51 orthodontists were asked about their preferences for the following appliances: clear aligners (CA), lingual metallic brackets (LMB), polycrystalline and monocrystalline ceramic brackets, and buccal metallic brackets (BMB). The participants rated the importance of 17 potential reasons that would explain their choices. The reasons that contributed most to these preferences were identified. Non-parametric tests (Fisher's exact, $\chi^{2}$ and Mann-Whitney tests) and multivariate analyses (regression and discriminant analysis) were used to assess the data $(\alpha=0.05)$. Results: CA and BMB were the most chosen appliances by PP and orthodontists, respectively. LMB was the most rejected option among both groups of participants $(p<$ 0.001). Rates of the importance of pain/discomfort, smile esthetics, finishing details, and feeding/speech impairment showed the highest differences between PP and orthodontists $(p<0.0005)$. Discriminant analyses showed that individuals who considered treatment time and smile esthetics as more important were more likely to prefer $C A$, while those who prioritized finishing details and cost were more likely to choose BMB $(p<0.05)$. Conclusions: Reasons related to comfort and quality of life during use were considered as more important by $\mathrm{PP}$, while those related to the results and clinical performance of the appliances were considered as more relevant by orthodontists.
\end{abstract}

[Korean J Orthod 2021;51(2):115-125]

Key words: Patient preference, Orthodontic appliances, Orthodontists

Received June 19, 2020; Revised September 29, 2020; Accepted October 26, 2020.

Corresponding author: Margareth Maria Gomes de Souza.

Full Professor, Department of Pediatric Dentistry and Orthodontics, School of Dentistry, Federal University of Rio de Janeiro, Rua. Prof. Rodolpho Paulo Rocco, 325 - Cidade Universitária da Universidade Federal do Rio de Janeiro, Rio de Janeiro, RJ, 21941-617, Brazil.

Tel +55-21-3938-2015 e-mail margasouzaster@gmail.com

How to cite this article: Marañón-Vásquez GA, Barreto LSC, Pithon MM, Nojima LI, Nojima MCG, Araújo MTS, Souza MMG. Reasons influencing the preferences of prospective patients and orthodontists for different orthodontic appliances. Korean J Orthod 2021;51:115-125.

(C) 2021 The Korean Association of Orthodontists.

This is an Open Access article distributed under the terms of the Creative Commons Attribution Non-Commercial License (http://creativecommons.org/licenses/by-nc/4.0) which permits unrestricted non-commercial use, distribution, and reproduction in any medium, provided the original work is properly cited. 


\section{INTRODUCTION}

Trends in the use of orthodontic appliances change over time. Currently, options that generate greater satisfaction related to esthetics and comfort during use are probably the main requirements of patients seeking treatment. ${ }^{1,2}$ However, in comparison with conventional buccal metallic brackets (BMB), most of these alternatives still present limited efficacy for the correction of certain cases..$^{3-5}$ Considering that there is still no "ideal appliance," the differences in clinical effectiveness, ${ }^{3,4}$ related comfort, ${ }^{2,6}$ and probability of adverse effects ${ }^{7-9}$ among the available options must be assessed by both patients and orthodontists before making their choice.

Patient preference is a difficult issue to assess, since this may be influenced not only by factors directly related to therapy, but also by subjective factors inherent to the individual, such as previous experiences, attitudes, or beliefs about treatment. ${ }^{10,11}$ Some studies have demonstrated that patients show greater acceptability for the appliances they deem more esthetic. ${ }^{1,12}$ Nevertheless, it is very likely that other reasons also influence their choices. In addition, many of the recommendations offered by orthodontists could be biased by their preferences and prior training using specific devices, without providing patients with complete information about the advantages and disadvantages of all options available.

Some studies have attempted to determine patients' motivations to undergo orthodontic treatment. ${ }^{13,14}$ However, little is known about the reasons influencing their preferences for the available appliances, and how orthodontists could use this information to identify suitable treatments for each patient. Therefore, the objectives of this study were (1) to evaluate the preferences of prospective patients (PP) and orthodontists for different orthodontic appliances, (2) to compare their judgments about the importance of potential reasons influencing these preferences, and (3) to identify predictive variables contributing to the selection or rejection of specific orthodontic appliances.

\section{MATERIALS AND METHODS}

The Research Ethics Committee of the Clementino Fraga Filho University Hospital - Federal University of Rio de Janeiro (HUCFF-UFRJ) approved the protocol of this cross-sectional study (no. 3.182.753). The methodological design was based on a previously published paper by Leles et al. ${ }^{15} \mathrm{~A}$ sample size calculation was performed to compare proportions of two independent groups (two-tailed Fisher's exact test) in G*Power 3.1 based on the estimate that $30 \%$ of PP and $80 \%$ of orthodontists selected BMB as their preferred option, and $70 \%$ and $20 \%$, respectively, marked this appliance as their rejected option (pilot study, 10 participants per group). Considering a power of $90 \%$, significance level of $5 \%$, and allocation ratio of $1: 1$, at least 46 participants (23 per group) were required for each comparison.

Forty-nine consecutive individuals (PP, mean age $=$ $37.4 \pm 17.8 ; 14$ men, 35 women) seeking orthodontic treatment at the Graduate Clinic in Orthodontics of the Federal University of Rio de Janeiro between March and June 2019 were selected for the present study. Participants that received previous orthodontic treatment were excluded. Additionally, 51 orthodontists (mean age = $36.4 \pm 11.3$; 16 men, 35 women) affiliated to the Brazilian Association of Orthodontists - Rio de Janeiro, were also selected. All participants provided written informed consent.

A three-part questionnaire was developed. In the first part, data such as gender, age, economic classification (Class A to E), ${ }^{16}$ and smile/occlusion auto-perception (assessed on a 0-10 visual analogue scale) were collected. Subsequently, participants were shown standardized edited photographs (Figure 1) of five orthodontic appliances: clear aligners (CA), lingual metallic brackets (LMB), polycrystalline ceramic brackets (PCB), monocrystalline ceramic brackets (MCB), and BMB. These images were obtained from the same person simulating the use of the different appliances for standardization purposes. PP and orthodontists were asked about their knowledge or technical training on each of the evaluated appliances, respectively. Knowledge of PP about the appliances
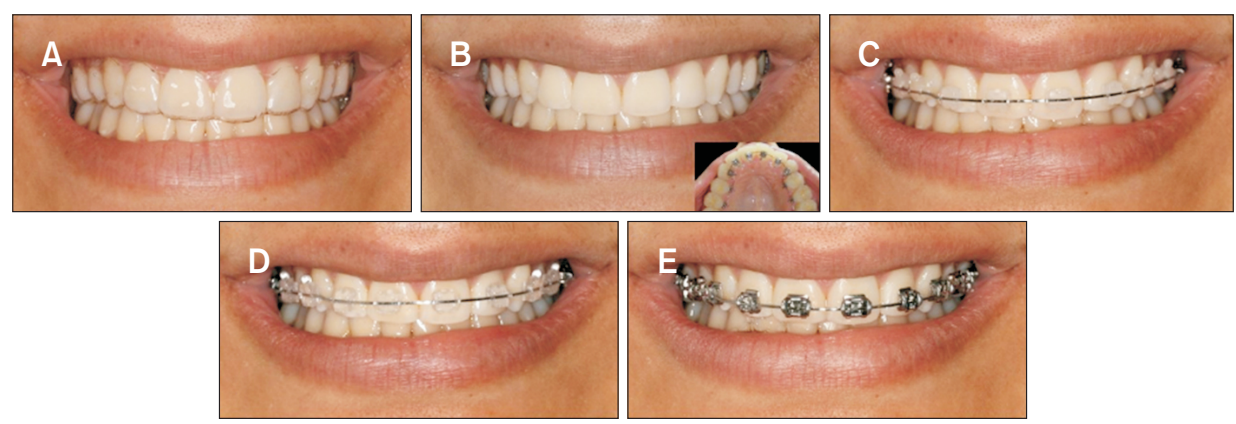

Figure 1. Images of the orthodontic appliances presented to the participants. $\mathbf{A}$, Clear aligners. B, Lingual metallic brackets. C, Polycrystalline ceramic brackets. D, Monocrystalline ceramic brackets. $\mathrm{E}$, Buccal metallic brackets. 
could be attributed to prior dental consultation with an orthodontist, media, or advertising.

In the second part, the participants were requested to rank these appliances in order of preference, on the premise that all of them solve the patient's malocclusion. Immediately after their responses, a printed chart (Table 1) with information about the advantages and disadvantages of the appliances was presented to the participants, while ensuring that the researcher could not influence the participants' perceptions of any of the appliances studied. To this end, the researcher was instructed to only answer participants' doubts in relation to a lack of understanding of the contents. Information for each of the appliances categorized under the following six items was shown to the participants: 1 - esthetics; 2 - treatment results; 3 - clinical performance; 4 - satisfaction, comfort, and quality of life; 5 - adverse effects; and 6 - cost of treatment (See the Supplementary Table 1 with the list of references considered for the contents of Table 1). Then, the participants were asked again to rank the appliances, now with equivalent information received (preferences finally used for analyses). The appliances ranked as number one and five were presumed as the chosen and refused treatments, respectively.

In the third part of the questionnaire, participants were requested to rate the importance of 17 potential reasons influencing their preferences (Figure 2). Questions were presented as previously described: ${ }^{17}$ How much do you consider __ to be an important reason for deciding about an orthodontic appliance? The par- ticipants recorded their responses using an ordinal fivepoint Likert scale $(1=$ no importance, $2=$ less important, $3=$ moderate importance, $4=$ very important, $5=$ extremely important).

Supplementary data is available at https://doi. org/10.4041/kjod.2021.51.2.115.

\section{Statistical analysis}

All analyses were performed using two-tailed tests ( $\alpha$ $=0.05$ ) in SPSS version 21.0 (IBM Corp., Armonk, NY, USA). Fisher's exact or $\chi^{2}$ tests were used for the following purposes: (1) to assess differences in previous knowledge of PP regarding the different appliances, and in technical training received by orthodontists, (2) to assess the influence of the provision of appliance-related information on the choice of individuals, and (3) to compare the chosen/refused treatment frequencies between PP and orthodontists, and among different orthodontic appliances. Regression analyses were performed to identify associations of factors (gender and economic classification) and covariates (age, smile and occlusion auto-perception), with the frequencies of choice of each appliance. Additionally, PP were grouped into a "high economic classification" group (economic classes A and B) and a "low economic classification" group (classes C, $\mathrm{D}$, and E). The $\chi^{2}$ test was applied to evaluate the association between a "high" or "low" economic classification and the type of appliance chosen.

Scores of PP and orthodontists were compared using Mann-Whitney test (ordinal variables). Discriminant analyses were used to create linear functions to identify

Table 1. Chart with appliance-related information

\begin{tabular}{|c|c|c|c|c|c|}
\hline Topic & CA & LMB & PCB & MCB & BMB \\
\hline Esthetics (Smile esthetics) & Very good & Very good & Good & Good & Bad \\
\hline $\begin{array}{l}\text { Treatment results (Results, finishing details, result } \\
\text { stability) }\end{array}$ & Medium & Good & Very good & Very good & Very good \\
\hline $\begin{array}{l}\text { Clinical performance (Ability of the appliance to obtain } \\
\text { results without difficulties and/or complications, and } \\
\text { in less time) }\end{array}$ & Medium & Good & Medium & Medium & Very good \\
\hline $\begin{array}{l}\text { Satisfaction, comfort, quality of life (Absence of pain/ } \\
\text { discomfort, no deterioration of oral functions, } \\
\text { less difficulty for oral hygiene, lower appointment } \\
\text { frequency, lower chair time, less chance of urgent } \\
\text { appointments) }\end{array}$ & Very good & Bad & Medium & Medium & Medium \\
\hline $\begin{array}{l}\text { Adverse effects (Lower probability of root resorption, } \\
\text { dental caries, gingivitis/periodontal disease, tooth } \\
\text { wear) }\end{array}$ & Very good & Good & Good & Good & Good \\
\hline Cost & Bad & Medium & Good & Medium & Very good \\
\hline
\end{tabular}

CA, clear aligners; LMB, lingual metallic brackets; $\mathrm{PCB}$, polycrystalline ceramic brackets; $\mathrm{MCB}$, monocrystalline ceramic brackets; BMB, buccal metallic brackets.

See the Supplementary Table 1 with the list of references considered for the contents. 


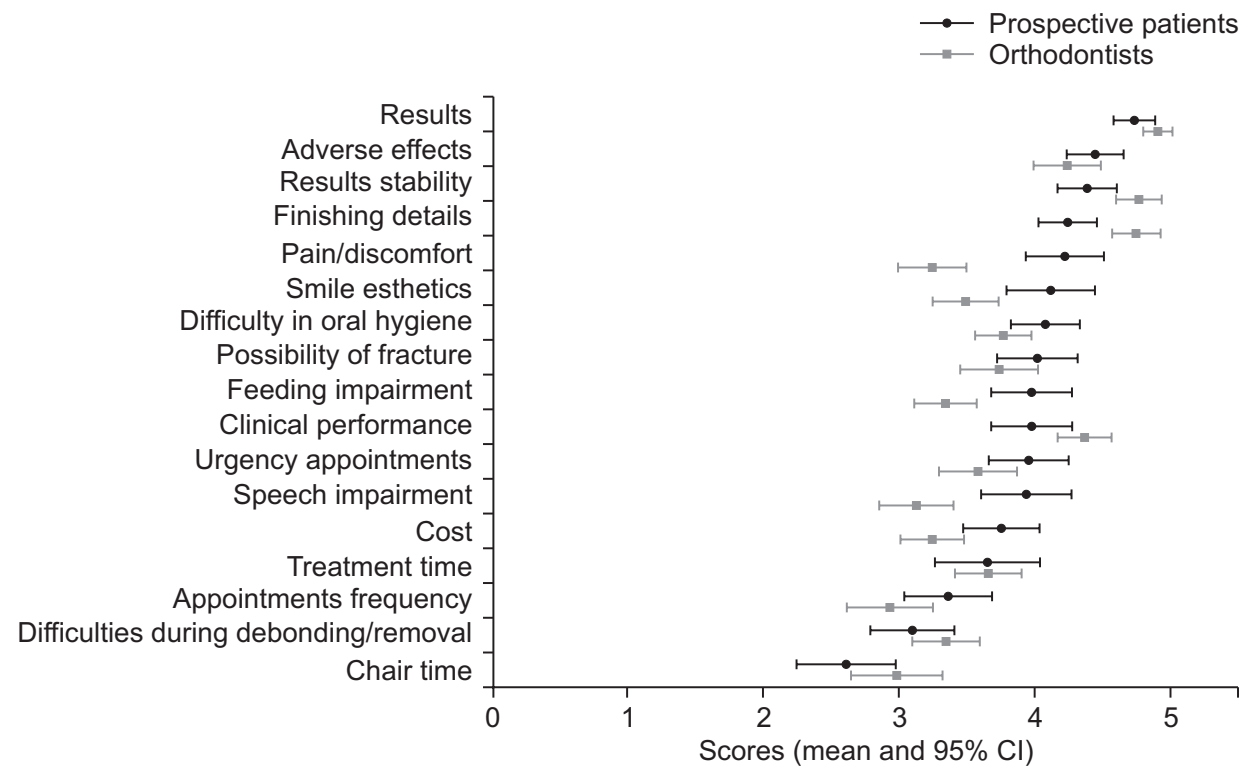

Figure 2. Scores recorded by prospective patients and orthodontists on the importance of the reasons influencing their preferences. The reasons are presented in order of importance to the prospective patients. The means and 95\% confidence intervals (Cls) are only representative values, since the medians (and interquartile ranges) were considered for statistical analysis (Graphic generated in GraphPad Prism [GraphPad Software, San Diego, CA, USA]).

1, no importance; 2, less important; 3, moderate importance; 4, very important; 5, extremely important.

Table 2. Previous knowledge/technical training on each of the assessed appliances

\begin{tabular}{lcccccc}
\hline \multicolumn{1}{c}{ Groups } & CA & LMB & PCB & MCB & BMB & p-value \\
\hline Prospective patients $(\mathrm{n}=49)$ & & & & & & $<0.001^{*}$ \\
$\quad$ Knowledge & $27(55.1)$ & $21(42.9)$ & $38(77.6)$ & $35(71.4)$ & $45(91.8)$ & \\
$\quad$ No knowledge & $22(44.9)$ & $28(57.1)$ & $11(22.4)$ & $14(28.6)$ & $4(8.2)$ & \\
Orthodontists $(\mathrm{n}=51)$ & & & & & & $<0.001^{*}$ \\
$\quad$ Technical training & $45(88.2)$ & $7(13.7)$ & $50(98.0)$ & $50(98.0)$ & $51(100.0)$ & \\
No technical training & $6(11.8)$ & $44(86.3)$ & $1(2.0)$ & $1(2.0)$ & $0(0.0)$ & \\
\hline
\end{tabular}

Values are presented as frequencies (\%).

Percentages are presents for the columns.

$\mathrm{CA}$, clear aligners; $\mathrm{LMB}$, lingual metallic brackets; $\mathrm{PCB}$, polycrystalline ceramic brackets; $\mathrm{MCB}$, monocrystalline ceramic brackets; BMB, buccal metallic brackets.

Chi-square test was performed.

${ }^{*} p<0.05$ indicates statistically significant association.

how these reasons (predictive/independent variables) contributed to the choice or rejection of the appliances evaluated (dependent variables). Within-group correlations of each predictor variable with the canonical function (structure matrix) were calculated to identify the most discriminant variables. Eigenvalue, canonical correlation, and Wilks' lambda were calculated to assess the predictive ability of the models. These were also validated by calculating the overall agreement after crosstabulation of original and predicted groups classifications.

\section{RESULTS}

Previous knowledge and technical training on each of the assessed appliances was significantly different for PP and orthodontists, respectively ( $p<0.001$; Table 2). In general, individuals were less familiar with LMB and more familiar with BMB. On the other hand, provision of information related to the advantages and disadvantages of orthodontic appliances significantly changed the opinions about the chosen/refused appliance in PP $(p<0.001)$, but not in orthodontists $(p>0.05)$ (Table 3$)$. 
Table 3. Chosen/refused appliances for prospective patients $(n=49)$ and orthodontists $(n=51)$, before and after receiving information on advantages and disadvantages of the assessed appliances

\begin{tabular}{|c|c|c|c|c|c|}
\hline \multirow{2}{*}{ Groups } & \multicolumn{2}{|c|}{ Pre-information } & \multicolumn{2}{|c|}{ Post-information } & \multirow{2}{*}{$p$-value } \\
\hline & Chosen & Refused & Chosen & Refused & \\
\hline \multicolumn{6}{|l|}{ Prospective patients } \\
\hline Clear aligners & $26(83.9)$ & $5(16.1)$ & $15(65.2)$ & $8(34.8)$ & 0.197 \\
\hline Lingual metallic brackets & $9(47.4)$ & $10(52.6)$ & $4(16.0)$ & $21(84.0)$ & $0.044^{*}$ \\
\hline Polycrystalline ceramic brackets & $4(66.7)$ & $2(33.3)$ & $10(90.9)$ & $1(9.1)$ & 0.515 \\
\hline Monocrystalline ceramic brackets & $5(55.6)$ & $4(44.4)$ & $7(100.0)$ & $0(0.0)$ & 0.089 \\
\hline Buccal metallic brackets & $5(15.2)$ & $28(84.9)$ & $13(40.6)$ & $19(59.4)$ & $0.028^{*}$ \\
\hline \multicolumn{6}{|l|}{ Orthodontists } \\
\hline Clear aligners & $17(85.0)$ & $3(15.0)$ & $19(82.6)$ & $4(17.4)$ & $>0.999$ \\
\hline Lingual metallic brackets & $0(0.0)$ & $44(100.0)$ & $0(0.0)$ & $43(100.0)$ & $>0.999$ \\
\hline Polycrystalline ceramic brackets & $2(100.0)$ & $0(0.0)$ & $2(100.0)$ & $0(0.0)$ & $>0.999$ \\
\hline Monocrystalline ceramic brackets & $3(100.0)$ & $0(0.0)$ & $2(100.0)$ & $0(0.0)$ & $>0.999$ \\
\hline Buccal metallic brackets & $29(87.9)$ & $4(12.1)$ & $28(87.5)$ & $4(12.5)$ & $>0.999$ \\
\hline
\end{tabular}

Values are presented as frequencies (\%).

Percentages are presents for the rows.

Fisher's exact test was performed.

${ }^{*} p<0.05$ indicates statistically significant association.

Table 4. Chosen/refused appliances for prospective patients and orthodontists

\begin{tabular}{|c|c|c|c|c|c|}
\hline \multirow{2}{*}{ Orthodontic appliances } & \multicolumn{2}{|c|}{ Prospective patients $(n=49)$} & \multicolumn{2}{|c|}{ Orthodontists $(n=51)$} & \multirow{2}{*}{$p$-value ${ }^{\dagger}$} \\
\hline & Chosen & Refused & Chosen & Refused & \\
\hline Clear aligners & $15(30.6)$ & $8(16.3)$ & $19(37.3)$ & $4(7.8)$ & 0.314 \\
\hline Lingual metallic brackets & $4(8.2)$ & $21(42.9)$ & $0(0.0)$ & $43(84.3)$ & $0.016^{*}$ \\
\hline Polycrystalline ceramic brackets & $10(20.4)$ & $1(2.0)$ & $2(3.9)$ & $0(0.0)$ & $>0.999$ \\
\hline Monocrystalline ceramic brackets & $7(14.3)$ & $0(0.0)$ & $2(3.9)$ & $0(0.0)$ & $>0.999$ \\
\hline Buccal metallic brackets & $13(26.5)$ & $19(38.8)$ & $28(54.9)$ & $4(7.8)$ & $<0.001^{*}$ \\
\hline$p$-value ${ }^{\ddagger}$ & \multicolumn{2}{|c|}{$<0.001^{*}$} & \multicolumn{2}{|c|}{$<0.001^{*}$} & \\
\hline
\end{tabular}

Values are presented as frequencies (\%).

Percentages are presented for the columns.

${ }^{*} p<0.05$ indicates statistically significant association.

${ }^{\dagger}$ Fisher's exact test was performed.

${ }^{*}$ Chi-square test was performed.

The chosen/refused frequencies for the LMB $(p=0.044)$ and BMB $(p=0.028)$ were significantly different after PP were informed (Table 3).

Comparisons of the frequencies of the final chosen/refused appliances for PP and orthodontists are presented in Table 4. Significant differences were found between PP and orthodontists in the frequencies of individuals choosing or refusing $\operatorname{LMB}(p=0.016)$ and BMB $(p<$ 0.001). Differences in the chosen/refused distributions between the different orthodontic appliances were significant $(p<0.001)$. While CA were more frequently chosen by $\mathrm{PP}, \mathrm{BMB}$ were more frequently chosen by orthodontists. LMB showed the highest prevalence of rejection for all participants. Regression analyses evidenced no associations between the factors or covariates assessed, and the frequencies of choice of each appliance. On the other hand, an association was observed between the economic classification and the preferred appliance ( $p=0.039$; Table 5); the most chosen option by the participants of the "high economic classification" group was CA, while for the participants of the "low economic classification" group, it was BMB. LMB was 
Table 5. Appliances chosen by prospective patients according to their economic classification

\begin{tabular}{|c|c|c|c|c|c|c|}
\hline \multirow[b]{2}{*}{ Economic classification } & \multirow[b]{2}{*}{ CA } & \multirow[b]{2}{*}{ PCB + MCB } & \multirow[b]{2}{*}{ BMB } & \multicolumn{3}{|c|}{$p$-value } \\
\hline & & & & $\begin{array}{c}\text { CA vs. } \\
\text { PCB + MCB }\end{array}$ & CA vs. BMB & $\begin{array}{l}\text { CA vs. } \\
\text { PCB + MCB vs. BMB }\end{array}$ \\
\hline High & $11(73.3)$ & $6(35.3)$ & $4(30.8)$ & \multirow{2}{*}{$0.031^{*}$} & \multirow{2}{*}{$0.024^{*}$} & \multirow{2}{*}{$0.039^{*}$} \\
\hline Low & $4(26.7)$ & $11(64.7)$ & $9(69.2)$ & & & \\
\hline
\end{tabular}

Values are presented as frequencies (\%).

Percentages are presented for the columns.

High economic classification includes Classes A and B. Low economic classification includes Classes C, D, and E.

$\mathrm{CA}$, clear aligners; $\mathrm{PCB}$, polycrystalline ceramic brackets; $\mathrm{MCB}$, monocrystalline ceramic brackets; $\mathrm{BMB}$, buccal metallic brackets.

Chi-square test was performed.

${ }^{*} p<0.05$ indicates a statistically significant association.

Table 6. Parameters providing information about the relative efficacy of the discriminant functions

\begin{tabular}{lcccc}
\hline Orthodontic appliances & Eigenvalue & $\begin{array}{c}\text { Canonical } \\
\text { correlation }\end{array}$ & Wilks' Lambda & p-value \\
\hline Clear aligners & & & & \\
Prospective patients & 12.022 & 0.961 & 0.077 & $0.015^{*}$ \\
Orthodontists & 2.863 & 0.861 & 0.259 & 0.462 \\
Total & 1.264 & 0.747 & 0.442 & $0.034^{*}$ \\
Buccal metallic brackets & & & & \\
Prospective patients & 1.429 & 0.767 & 0.412 & 0.324 \\
Orthodontists & 2.064 & 0.821 & 0.326 & 0.117 \\
Total & 0.997 & 0.707 & 0.501 & $0.003^{*}$ \\
\hline
\end{tabular}

Total includes prospective patients and orthodontists.

${ }^{*} p<0.05$ indicates functions showing the best predictive ability.

not part of this analysis due to the low frequency of this appliance as the chosen option. PCB and MCB were included into one single category (ceramic brackets) to enable these analyses.

Scores recorded by the PP and orthodontists for each reason assessed are presented in Figure 2. Reasons judged as the most important (scores 4 and 5; with frequency $\geq 35$ ) for PP were results, adverse effects, result stability, finishing details, difficulty in oral hygiene, smile esthetics and pain/discomfort, while for orthodontists, they were results, result stability, clinical performance, finishing details, and adverse effects. The reasons showing large differences in the scores rated between the groups were as follows: pain/discomfort, smile esthetics $(p<0.0001)$, finishing details, feeding, and speech impairment $(p<0.0005)$. Although significant, less difference was presented for result stability, cost $(p<0.005)$, difficulty in oral hygiene, appointment frequency, and possibility of urgency appointments $(p<0.05)$.

Because of the low frequencies of chosen or refused options for $\mathrm{LMB}, \mathrm{PCB}$, and $\mathrm{MCB}$, these appliances were not included in the discriminant analysis. Information related to the relative efficacy of each discriminant function is presented in Table 6 . Functions evidencing $p$ $<0.05$ showed predictive ability to identify participants' preferences based on the rated importance for the reasons studied. Reasons with a higher impact for choosing or refusing CA and BMB are presented in Table 7. Individuals who considered treatment time and smile esthetics as reasons of greater importance are more likely to prefer CA while for those who prioritized results and cost of the treatment, the probability of refusing this appliance is greater. On the other hand, while participants who prioritize reasons such as finishing details and cost of the treatment are more likely to choose BMB, those who consider smile esthetics and general discomfort (feeding and speech impairment, and pain/discomfort) as being of greater importance are more likely to reject this appliance. The overall agreements for validation of the models ranged from $85.9-100 \%$.

\section{DISCUSSION}

Current orthodontic practice presumes clinician- 
Table 7. Predictive variables and structural matrix of the discriminant functions for choosing or refusing clear aligners and buccal metallic brackets

\begin{tabular}{llcll}
\hline Orthodontic appliances & Prospective patients & Structure matrix & \multicolumn{1}{c}{ Total } & Structure matrix \\
\hline Clear aligners & Choosing & & Choosing & \\
& Treatment time & 0.180 & Smile esthetics & 0.467 \\
& Smile esthetics & 0.167 & Treatment time & 0.371 \\
& & & Difficulty in oral hygiene & 0.327 \\
& & & Urgency appointments & 0.242 \\
& & & Appointments frequency & 0.215 \\
& & & Adverse effects & 0.204 \\
& Refusing & & Refusing & \\
& Possibility of fracture & 0.500 & Cost & 0.630 \\
& Results & 0.210 & Results & 0.580 \\
Buccal metallic brackets & Choosing & & Choosing & \\
& Finishing details & 0.388 & Finishing details & 0.367 \\
& Cost & 0.333 & Chair time & 0.246 \\
& & & Cost & 0.202 \\
& & & Results & 0.192 \\
& Refusing & & Refusing & \\
& Feeding impairment & 0.347 & Smile aesthetics & 0.464 \\
& Smile esthetics & 0.214 & Feeding impairment & 0.400 \\
& Treatment time & 0.155 & Pain/discomfort & 0.324 \\
& & Speech impairment & 0.230 \\
& & & Adverse effects & 0.175 \\
& & & Possibility of fracture & 0.160 \\
\hline
\end{tabular}

Total includes prospective patients and orthodontists.

Structural matrix is presented as absolute values, ordered by the size of correlation within functions. Only coefficients $>0.15$ are shown.

patient interaction in the determination of problemoriented diagnosis and treatment planning. In that context, it is important to determine the preferences of the patients to make recommendations that adequately guide decision-making. To the best of our knowledge, this is the first study comparing the preferences of PP and orthodontists for different types of orthodontic appliances and evaluating the reasons that each consider to be important for their choice.

Previous studies performed to assess the preferences of patients for different orthodontic devices based on their attractiveness, demonstrated, with relative consistency, that CA or LMB are the most widely accepted alternatives. ${ }^{1,12,18}$ In the present study, similarly, when PP were asked about their preference only by observing images of the devices (before being informed), CA and LMB were the first and second preferred options, respectively, while BMB was the most rejected option. Interestingly, LMB was also the second-most rejected option, which could be due to the limited knowledge and uncertainty of the patients regarding these appliances (more than half of PP reported not having any prior knowledge about LMB). These results confirm that, apparently, the esthetics offered by the devices is perhaps the most influential reason for the patients' decision when they do not receive any other appliance-related information. This assumption was supported by the result that for PP, smile esthetics was rated as one of the most important reasons when choosing some types of appliances. The individuals who considered this aspect as of great importance were more likely to prefer CA or to reject BMB. Indeed, it has been shown that the use of buccal brackets could negatively change the patients' self-perception of their beauty (during use), which may affect their selfesteem, and consequently, their social relationships. ${ }^{19} \mathrm{Al}-$ though this information is controversial, it makes sense to think that the first thing most people will look for is an appliance that does not adversely affect their appear- 
ance.

After receiving information about the advantages and disadvantages of the evaluated appliances, even though $\mathrm{CA}$ remained the most chosen option, the distribution of PP preference changed. BMB was the second most chosen option, showing that many patients value other aspects of the devices in addition to its esthetics. BMB has generally been shown to achieve the best treatment results. Although the evidence is still insufficient, of low quality and heterogeneous, CA has been reported to be a viable alternative mainly for mild to moderate malocclusions in non-growing patients that do not require extractions, but they still do not achieve the same effectiveness as BMB for some types of orthodontic movements. ${ }^{3,20}$ Furthermore, even though CA have been shown to be effective, they may not achieve as detailed and stable results as BMB. ${ }^{21}$ In the present study, the reasons rated as important by $\mathrm{PP}$ are consistent with their preferences. Among the reasons that participants judged to be most important are results, result stability, and finishing details. Individuals who rated finishing details as of great importance were more likely to prefer $\mathrm{BMB}$, and those who considered results as of great importance were more likely to reject CA. On the other hand, a considerable percentage of PP chose PCB or $\mathrm{MCB}$ as the preferred option, suggesting that individuals could decrease their esthetic requirements, to a certain extent, in the attempt to obtain results more similar to those obtained with BMB. Similarly, since CA is the alternative that offers less pain and greater satisfaction during use, ${ }^{2,22}$ these changes in preferences would demonstrate that they could also sacrifice this requirement for obtaining better results. Apparently, the smile esthetics, comfort, and results offered by the appliances would be extremely important factors in making a decision.

For the LMB, previous studies have reported that, although these show some biomechanical limitations, these can achieve very similar results to those obtained with BMB. ${ }^{4,23}$ However, these appliances have been associated with increased oral discomfort, impaired speech performance, and increased difficulty in eating. ${ }^{4,24,25}$ Moreover, patients using these devices have been reported to present greater problems in maintaining proper oral hygiene. ${ }^{7}$ These disadvantages could be the reason why for $\mathrm{PP}, \mathrm{LMB}$ was the most rejected option after being informed. The PP reported adverse effects, difficulty in oral hygiene, and pain/discomfort as the most important reasons for their preference. To a lesser extent, BMB can also cause some pain or discomfort in the cheek and lip, ${ }^{24}$ and eating difficulties. ${ }^{6}$ This would explain why these appliances were also rated as the second most rejected option. Participants who prioritized general discomfort (feeding and speech impairment and pain/discomfort) as being of greater importance were more likely to reject $\mathrm{BMB}$.

It is also important to consider that preferences, and the reasons that motivate them, are likely to change depending on the sample that is assessed. Some evidence suggests that the economic status could influence patients' preferences. One study showed that patients who used CA had a significantly higher income than those treated with fixed appliances. ${ }^{26}$ Likewise, patients may be willing to pay more money for appliances they deem more esthetic. ${ }^{1}$ Our results agree with this evidence; being of higher economic class was associated with choosing CA, while being of lower economic class was associated with choosing BMB or any of the ceramic bracket alternatives. Further studies should be conducted with larger samples stratified according to economic class to evaluate differences in preferences according to this factor. On the other hand, although the present study did not show the influence of gender, age, and self-perception of the smile/occlusion on the preferences of the participants, it suggested that future studies also consider these variables, since they could also influence decision-making.

Divergences exist in treatment preferences between patients and professionals from different areas of health. ${ }^{27}$ There are no previous studies evaluating this issue in orthodontics. Since orthodontists have a contrasting view to that of patients on the need for treatment, ${ }^{28}$ their appliance preferences are also different. Our results demonstrated significant differences between the preferences of PP and orthodontists. Although LMB was the most rejected option in both groups, the percentage was considerably higher for orthodontists (more than 80\% of orthodontists rejected this option). We consider that this result was due to the fact that a similar percentage of specialists reported not having received prior technical training for the use of this device. This limitation of the specialty of orthodontics has already been previously reported $^{29}$; most orthodontists do not use LMB even when they offer clear biomechanical and esthetic advantages because the majority of specialists are not educated and trained to use these appliances. On the other hand, the most chosen option by orthodontists was BMB. The preferences for this type of appliance were significantly different from that of the PP. Among the reasons that this group deemed most important are results, result stability, and finishing details. This would suggest that orthodontists prioritize results at the time of their selection. However, this preference may also have been influenced by the prior knowledge this group had about BMB. All orthodontists were technically trained in the use of this device. It is obvious to think that the greater familiarity and confidence in the use of BMB made this option the most chosen and, perhaps, most recommended by them. Clinical performance was another 
reason that orthodontists rated as most important. This could explain why PCB and MCB had very low frequencies as chosen options. Indeed, these devices have been reported to show certain biomechanical limitations during use. ${ }^{5}$ Importantly, CA was the second most chosen option by orthodontists, which could be explained by two reasons deemed important for this group: clinical performance and adverse effects. Despite the fact that the treatment results of CA are not as good as those of $\mathrm{BMB},{ }^{3,20,21}$ these devices allow orthodontic mechanics with few complications. This can be evidenced from the fact that the number of emergency visits and emergency chair time for CA were significantly less than those for edgewise brackets. ${ }^{30}$ On the other hand, for adverse effects, the use of CA reduces periodontal damage ${ }^{9}$ as well as the incidence and severity of root resorption that is caused by traditional orthodontic therapies. ${ }^{8}$

Based on the pattern of responses of PP, it is highly likely that initial preferences were based on the visual impact and attractiveness of each appliance and the participants' prior knowledge of these. On the other hand, our findings demonstrated that, evidently, the participants change their opinions once they were informed on the particularities of the devices. It is important to note that the classifications (judgments) presented in Table 1 (i.e., very good, good, medium, bad), although supported by relevant evidences (Supplementary Table 1), were established by the authors. When the available evidence on a certain topic was controversial (inconsistent results between the studies), insufficient, or absent, the authors determined the classification for the appliance based on their clinical experience (e.g., in the case of the cost of treatments). This form of classification could have introduced a certain risk of bias associated with the authors' preferences, mainly for cases in which the information was insufficient. Fortunately, for most of the studied aspects, the literature showed a consistent pattern of results. It should also be mentioned that some factors such as the severity of the case and the clinical ability of the orthodontist using the different orthodontic appliances could modify the judgments presented in Table 1. In the present study, in order to control these factors, the preferences of the participants were evaluated under the premise that all appliances would be able to resolve their malocclusion (or the patient's malocclusion, for orthodontists). Since, in actual clinical practice, there is a possibility of variation in relation to these factors (among others, such as the type, design, brand, or prescription for each appliance), the reported preferences should be evaluated according to the context studied. Future studies should be carried out with new information provided to the participants, since there is a constant evolution of orthodontic appliances. The changes in the PP preferences after provision of information demonstrate the importance of patientorthodontist communication in decision-making. ${ }^{11}$ Although the evidence outlining how patients make sense of orthodontic problems and treatments is scarce, knowledge of the reasons why the patient is considering such a treatment could be a starting point. It will be the task of the orthodontist to identify these reasons and determine how to properly guide the patient in their selection, which will also depend on the diagnosis and severity of the case.

Most of the reasons evaluated were rated as having some degree of importance for both study groups; however, some presented significant differences. In general, reasons related to comfort and quality of life during the use were considered as more important by PP than by orthodontists; reasons related to the results and clinical performance of the appliances were considered as more important by orthodontists than by PP. These results have great applicability for clinical practice, since they will allow the implementation of marketing processes according to the demands and preferences of patients, improve the information strategies provided for different alternatives of appliances, and to plan targeted treatments according to the need/demand of each patient. As a specialist, the orthodontist must understand that a certain type of appliance will be an alternative for some cases and not for others.

\section{CONCLUSION}

The preferences of PP and orthodontists were different. More than orthodontists, PP considered reasons related to the comfort and quality of life during use to identify a certain type of appliance; the reasons related to the results and clinical performance of the appliances were considered more important by orthodontists than by PP.

\section{CONFLICTS OF INTEREST}

No potential conflict of interest relevant to this article was reported.

\section{ACKNOWLEDGEMENTS}

This study was supported in part by the Coordination for the Improvement of Higher Education Personnel (CAPES, Brazil) - Financing code: 001.

The authors express their gratitude to the patients who sought dental care in the Department of Pediatric Dentistry and Orthodontics of the Federal University of Rio de Janeiro, as well as to orthodontists affiliated to the Brazilian Association of Orthodontists - Rio de Janeiro, for their participation and time offered to answer 
the questionnaires.

\section{REFERENCES}

1. Rosvall MD, Fields HW, Ziuchkovski J, Rosenstiel SF, Johnston WM. Attractiveness, acceptability, and value of orthodontic appliances. Am J Orthod Dentofacial Orthop 2009;135:276.e1-12; discussion 276-7.

2. White DW, Julien KC, Jacob H, Campbell PM, Buschang $\mathrm{PH}$. Discomfort associated with Invisalign and traditional brackets: a randomized, prospective trial. Angle Orthod 2017;87:801-8.

3. Papadimitriou A, Mousoulea S, Gkantidis N, Kloukos D. Clinical effectiveness of Invisalign ${ }^{\circledR}$ orthodontic treatment: a systematic review. Prog Orthod 2018; 19:37.

4. Papageorgiou SN, Gölz L, Jäger A, Eliades T, Bourauel C. Lingual vs. labial fixed orthodontic appliances: systematic review and meta-analysis of treatment effects. Eur J Oral Sci 2016;124:105-18.

5. Reicheneder CA, Baumert U, Gedrange T, Proff P, Faltermeier A, Muessig D. Frictional properties of aesthetic brackets. Eur J Orthod 2007;29:359-65.

6. Shalish M, Cooper-Kazaz R, lvgi I, Canetti L, Tsur B, Bachar E, et al. Adult patients' adjustability to orthodontic appliances. Part 1: a comparison between Labial, Lingual, and Invisalign ${ }^{\mathrm{TM}}$. Eur J Orthod 2012; 34:724-30.

7. Ata-Ali F, Ata-Ali J, Ferrer-Molina M, Cobo T, De Carlos F, Cobo J. Adverse effects of lingual and buccal orthodontic techniques: a systematic review and meta-analysis. Am J Orthod Dentofacial Orthop 2016;149:820-9.

8. Fang X, Qi R, Liu C. Root resorption in orthodontic treatment with clear aligners: a systematic review and meta-analysis. Orthod Craniofac Res 2019; 22:259-69.

9. Jiang Q, Li J, Mei L, Du J, Levrini L, Abbate GM, et al. Periodontal health during orthodontic treatment with clear aligners and fixed appliances: a metaanalysis. J Am Dent Assoc 2018;149:712-20.e12.

10. Leles CR, Freire Mdo C. A sociodental approach in prosthodontic treatment decision making. J Appl Oral Sci 2004;12:127-32.

11. Broder HL, Phillips C, Kaminetzky S. Issues in decision making: should 1 have orthognathic surgery? Semin Orthod 2000;6:249-58.

12. Walton DK, Fields HW, Johnston WM, Rosenstiel SF, Firestone AR, Christensen JC. Orthodontic appliance preferences of children and adolescents. Am J Orthod Dentofacial Orthop 2010;138:698.e1-12; discussion 698-9.

13. McKiernan EX, McKiernan F, Jones ML. Psychological profiles and motives of adults seeking orthodon- tic treatment. Int J Adult Orthodon Orthognath Surg 1992;7:187-98.

14. de Souza RA, de Oliveira AF, Pinheiro SM, Cardoso JP, Magnani MB. Expectations of orthodontic treatment in adults: the conduct in orthodontist/patient relationship. Dental Press J Orthod 2013;18:88-94.

15. Leles CR, Martins RR, Silva ET, Nunes MF. Discriminant analysis of patients' reasons for choosing or refusing treatments for partial edentulism. J Oral Rehabil 2009;36:909-15.

16. Associação Brasileira de Empresas de Pesquisa. Critério de classificação econômica Brasil [Internet]. São Paulo: Associação Brasileira de Empresas de Pesquisa; 2018 Apr 16 [cited 2018 Oct 22]. Available from: http://www.abep.org/criterioBr/01_cceb_2018.pdf.

17. Leles CR, Ferreira NP, Vieira AH, Campos AC, Silva ET. Factors influencing edentulous patients' preferences for prosthodontic treatment. J Oral Rehabil 2011;38:333-9.

18. Ziuchkovski JP, Fields HW, Johnston WM, Lindsey DT. Assessment of perceived orthodontic appliance attractiveness. Am J Orthod Dentofacial Orthop 2008;133(4 Suppl):S68-78.

19. Fonseca LM, Araújo TM, Santos AR, Faber J. Impact of metal and ceramic fixed orthodontic appliances on judgments of beauty and other facerelated attributes. Am J Orthod Dentofacial Orthop 2014;145:203-6.

20. Robertson L, Kaur H, Fagundes NCF, Romanyk D, Major P, Flores Mir C. Effectiveness of clear aligner therapy for orthodontic treatment: a systematic review. Orthod Craniofac Res 2020;23:133-42.

21. Kuncio D, Maganzini A, Shelton C, Freeman K. Invisalign and traditional orthodontic treatment postretention outcomes compared using the American Board of Orthodontics objective grading system. Angle Orthod 2007;77:864-9.

22. Cardoso PC, Espinosa DG, Mecenas P, Flores-Mir C, Normando D. Pain level between clear aligners and fixed appliances: a systematic review. Prog Orthod 2020;21:3.

23. Mistakidis I, Katib H, Vasilakos G, Kloukos D, Gkantidis N. Clinical outcomes of lingual orthodontic treatment: a systematic review. Eur J Orthod 2016; 38:447-58.

24. Long H, Zhou Y, Pyakurel U, Liao L, Jian F, Xue J, et al. Comparison of adverse effects between lingual and labial orthodontic treatment. Angle Orthod 2013;83:1066-73.

25. Chen J, Wan J, You L. Speech and orthodontic appliances: a systematic literature review. Eur J Orthod 2018;40:29-36.

26. Miller KB, McGorray SP, Womack R, Quintero JC, Perelmuter M, Gibson J, et al. A comparison of 
treatment impacts between Invisalign aligner and fixed appliance therapy during the first week of treatment. Am J Orthod Dentofacial Orthop 2007; 131:302.e1-9.

27. Montgomery AA, Fahey T. How do patients' treatment preferences compare with those of clinicians? Qual Health Care 2001;10 Suppl 1(Suppl 1):i39-43.

28. Cai Y, Du W, Lin F, Ye S, Ye Y. Agreement of young adults and orthodontists on dental aesthetics \& in- fluencing factors of self-perceived aesthetics. BMC Oral Health 2018;18:113.

29. Riolo C. Lingual orthodontics: adding value to the care we offer our patients. Semin Orthod 2018; 24:P269-70.

30. Buschang PH, Shaw SG, Ross M, Crosby D, Campbell PM. Comparative time efficiency of aligner therapy and conventional edgewise braces. Angle Orthod 2014;84:391-6. 